\title{
Preparation of 5-fluorouracil loaded chitosan microparticle and its drug release properties
}

\author{
Mingming $\mathrm{Li}^{\mathrm{a}}$, Shiping Zhan ${ }^{\mathrm{b}}$ \\ Department of Environment and Chemical Engineering, Dalian University, Dalian, China
}

\begin{abstract}
Chitosan is one kind of good biocompatible polymer and is suitble for drug carriers. Preparation of 5-fluorouracil (5-Fu) loaded chitosan (CS) particles and in vitro release experiment were performed using ionic crosslinking method with sodium tripolyphosphate (TPP) as crosslinker. The optimal preparing parameters were verified by 5-Fu release experiments. The drug loading, and release behavior of drug loaded microparticles in vitro were investigated. The optimal preparation conditions were: the temperature $25^{\circ} \mathrm{C}$, the ratio of CS to TPP $5: 1$, the CS concentration $1.5 \mathrm{~g} / \mathrm{L}$, stirring speed $650 \mathrm{rpm}$. Under these conditions, the drug loading of particles was up to $45 \%$.
\end{abstract}

\section{Introduction}

5-Fluorouracil (5-Fu), an analogue of the natural pyrimidine uracil, is an anticancer drug that has been widely used in the management of solid malignancies arising from breast, gastrointestinal tract, head and neck, and colorectal cancer in particular ${ }^{[1-4]} .5-\mathrm{Fu}$ is having lack of site specificity and poor therapeutic effect. Also toxic effects to healthy cells and unavailability of major proportion of drug at the colon region remain as limitations ${ }^{[5]}$. To reduce its adverse effects, several efforts have been made to improve the delivery and the bioavailability of $5-\mathrm{Fu}$. The controlled drug delivery system of the drug-loaded polymer provides an alternative nonsurgical treatment for cancer. Moreover, the advantage of the drug-loaded micropaticles as drug delivery system is that this system can be maintained effective dosage longer than that with simple solution of the drug in lesion location ${ }^{[6,7]}$.

Chitosan (CS), a kind of natural polysaccharide, is widely used as drug carrier, due to its good biocompatibility and biodegradability. Drug loaded with chitosan microspheres can get to the Tumor targeted area and control the slow-release of the drug. Thereby, it can reduce the toxic and side effects of drugs on normal tissues.

This work was aimed to prepare 5-Fu loaded chitosan microparticles for sustained delivery application with ionic crosslinking method. The effects of preparing parameters on the drug loading of particles and release characteristic were studied.

\section{Materials and methods}

\subsection{Materials}

\footnotetext{
${ }^{\text {a }}$ Corresponding author: limingming@dlu.edu.cn

${ }^{\mathrm{b}}$ Corresponding author: zhanshiping@dlu.edu.cn
} 
5-Fu (purity $>98 \%$ ), used as active pharmaceutical ingredient, was purchased from Zhuorui Chem. (Shanghai, China). CS (purified), used as polymer was obtained from Haiyun Chem. (Dalian, China). Sodium tripolyphosphate(TPP, purified), used as crosslinker was obtained from Tianjin Kemiou Chemical Reagent Co.(Tianjin, China). Phosphate buffer saline (PBS) tablets (pH 6.8), which were used to prepare the drug release medium, were obtained from Tianjin Damao Chemical reagent Factory (Tianjin, China). Dialysis bag was perchused from Beijing JingKeHongDa (Beijing, China). All of the reagents were used without any further purification.

\subsection{Preparation of 5-Fu loaded chitosan microparticles}

The method used for the preparation of chitosan microparticles containing 5-FU is ionic crosslinking technique. Different CS concentrations $(0.6 \mathrm{~g} / \mathrm{L}, 0.8 \mathrm{~g} / \mathrm{L}, 1.0 \mathrm{~g} / \mathrm{L}, 1.5 \mathrm{~g} / \mathrm{L}, 2.0 \mathrm{~g} / \mathrm{L}, 2.5 \mathrm{~g} / \mathrm{L})$ was selected in order to optimize the best one and also to observe the effect of polymer on the formulation. Acetic acid was used as organic solvent with the concentration of $0.1 \%(\mathrm{w} / \mathrm{v})$. Drug was dissolved in acetic acid with varying polymer concentrations. Then it was followed by addition of TPP with varying ratio of CS to TPP $(3: 1,4: 1,5: 1,6: 1,8: 1)$, and was stirred continuously for $1.5 \mathrm{~h}$ with different stirring rates $(650 \mathrm{rpm}, 750 \mathrm{rpm}, 850 \mathrm{rpm}, 950 \mathrm{rpm}, 1050 \mathrm{rpm})$. Then the suspension was subjected to centrifugation. Supernatant was removed and washed repeatedly three times. Microparticles were obtained after drying in a vacuum oven.

\subsection{Drug loading}

Microparticles after drying were added to their specific solvent to facilitate the coat of the microparticles to get dissolved. The resultant suspension was subjected to evaporation for further removal of the solvent prior to filtration. Then the residue was washed and diluted appropriately with phosphate buffer of pH6.8 to determine drug content and entrapment efficiency. Samples were measured at an absorbance of $265 \mathrm{~nm}$ in ultraviolet-spectrophotometer (UV; 756PC, Shanghai Spectrum Instument Co., Ltd. Shanghai, China). Drug content loading of 5-Fu in CS microparticles was determined by the following equation ${ }^{[8]}$ :

$$
\text { Drug loading content }(\% w / w)=\frac{\text { Weight of drug in microparticle }}{\text { Weight of microparticle recovered }} \times 100
$$

\subsection{In Vitro experiment of drug release}

In drug release test, approximately $20 \mathrm{mg}$ sample was weighed and filled into a dialysis bag which was placed into a conical flask containing $30 \mathrm{~mL}$ of PBS (pH 6.8). Then, the conical flask was placed in a shaking incubator (SHZ-82; FeiPu Experimental Instrument Factory, Changzhou, China) at 100rpm and $37^{\circ} \mathrm{C}$ to simulate physiological conditions. At sampling points, $3 \mathrm{~mL}$ of the $\mathrm{PBS} /$ drug solution was removed from the flask and a fresh PBS solution with an identical volume was added to the maintain sink conditions. The $5-\mathrm{Fu}$ in the sample was measured at $265 \mathrm{~nm}$ with an ultraviolet-spectrophotometer. The release amount of 5-Fu was calculated with a predetermined standard curve $(\mathrm{C}=18.6916 \mathrm{~A}$ $\left.0.6093, R^{2}=0.9996\right)$.

\section{Results and discussions}

\subsection{Drug loading studies}

Figure 1 shows the effect of CS concentration on the drug loading of 5-Fu loaded CS microparticles. The drug loading increases with the increasing CS concentration, and achieves the highest amount of 
nearly $50 \%$ at $1.5 \mathrm{~g} / \mathrm{L}$ CS concentration. Then the drug loading decreases with the rising CS concentration. As long as the CS concentration increase, the reaction time gets longer, which leads to the more adsorption of 5-Fu onto the microparticles.

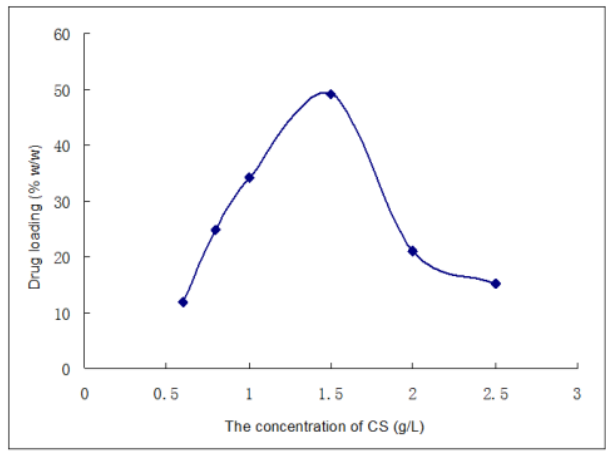

Figure 1. The effect of CS concentration on the drug loading of 5-Fu loaded CS microparticles

Figure 2 shows the effect of CS to TPP ratio on the drug loading of 5-Fu loaded CS microparticles. As shown in Figure 2, the amount of drug loading changes more evidently with the increase of ratio of CS to TPP. When the ratio is $5: 1$, the drug loading is about $34(\%, \mathrm{w} / \mathrm{w})$

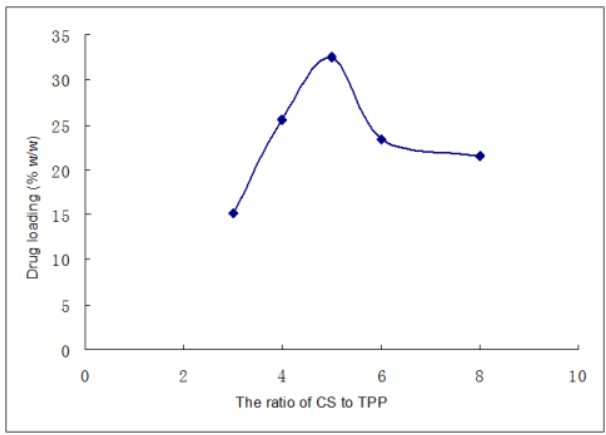

Figure 2. The effect of CS to TPP ratio on the drug loading of 5-Fu loaded CS microparticles

Figure 3 shows the effect of stirring rate on the drug loading of 5-Fu loaded CS microparticles. As shown in Figure 3, the drug loading presents a obviously decreases with the increase of stirring rate. When the rate is $650 \mathrm{rpm}$, the drug loading is about $25(\% \mathrm{w} / \mathrm{w})$. When the stirring rate is too fast, the vigorous stirring leads to the agglomeration of the dispersed microparticles, hence to the decreasing of 5-Fu drug loading.

\subsection{Drug release studies}

Figure 4 shows the 5-Fu release profiles from the CS microparticles with different CS concentrations. The release is completed in $12 \mathrm{~h}$ under PBS solution ( $\mathrm{pH} 6.8$ ) condition, although the test continued for $24 \mathrm{~h}$ in order to ensure that no more drug released at the end of the test. Total amount of impregnation mass is assumed to correspond to the accumulated drug released at the end of the test. The release studies show that, in the case with $1.0 \mathrm{~g} / \mathrm{L} \mathrm{CS}$ concentration, the cumulative release rate is highest. 


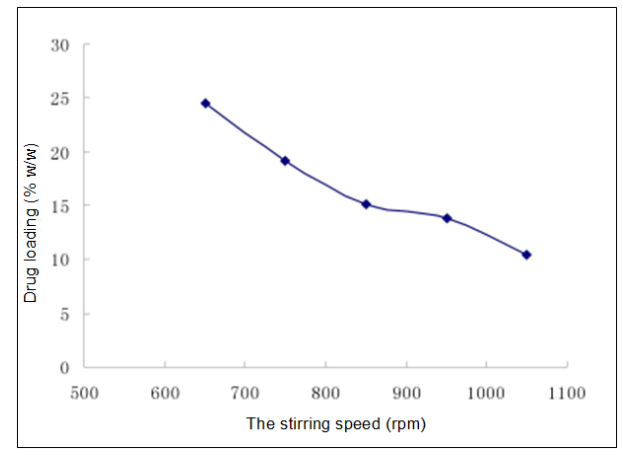

Figure 3. The effect of stirring rate on the drug loading of 5-Fu loaded CS microparticles

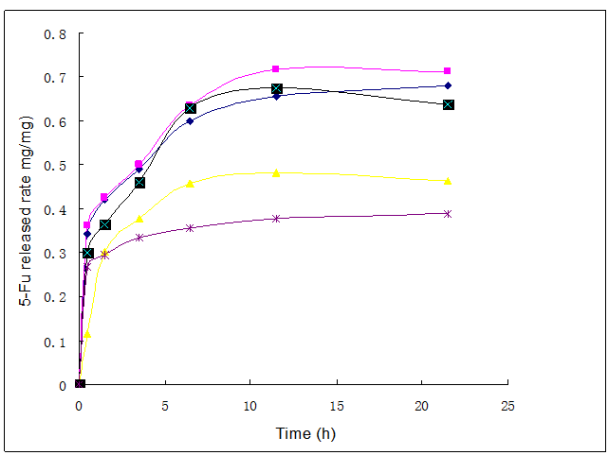

Figure 4. 5-Fu released rate from CS microparticles, with different CS concentrations $(\mathrm{g} / \mathrm{L})$ : $(\bullet) 0.8 ;(\mathbf{\bullet}) 1.0 ;(\boldsymbol{\Delta})$ $1.5 ;(\times) 2 ;(*) 2.5$

Figures 5 show the effects of ration of CS to TPP on the impregnation efficiencies. As shown in the curves, the ratio of CS to TPP plays an importent role in the 5-Fu release rate. In all the range of ratios, the drug release shows the same variation trend. In the first $6 h$, the release rate increase dramatically while the curve increase slowly in the next $6 \mathrm{~h}$. Then, the release rate remains smoothly during the last $12 \mathrm{~h}$. When the ratio of CS to TPP is $8: 1$, the 5-Fu release rate is highest with the amount of about $0.7 \mathrm{mg} / \mathrm{mg}$.

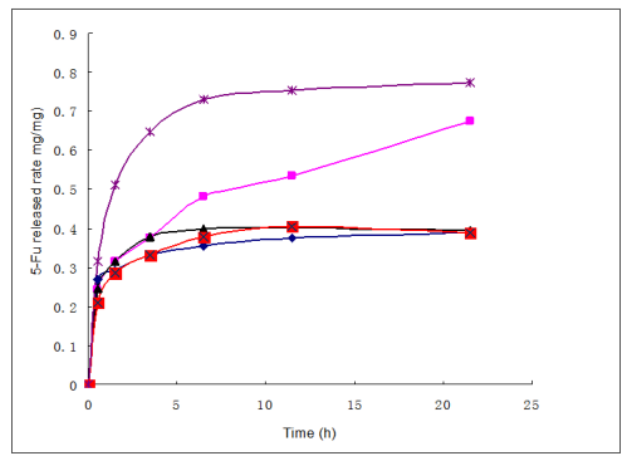

Figure 5. 5-Fu released rate from CS microparticles, with different ratio of CS to TPP: (४) $3: 1 ;(\mathbf{\square}) 4: 1 ;(\mathbf{\Lambda})$ $5: 1 ;(\times) 6: 1 ;(*) 8: 1$ 
Figures 6 show the effects of the stirring rate on the 5-Fu release rate. As shown in the Figures 6 , when the stirring rate is $950 \mathrm{rpm}$, the drug release rate can achieve the highest level, which is about $0.9 \mathrm{mg} / \mathrm{mg}$ in the first $12 \mathrm{~h}$.

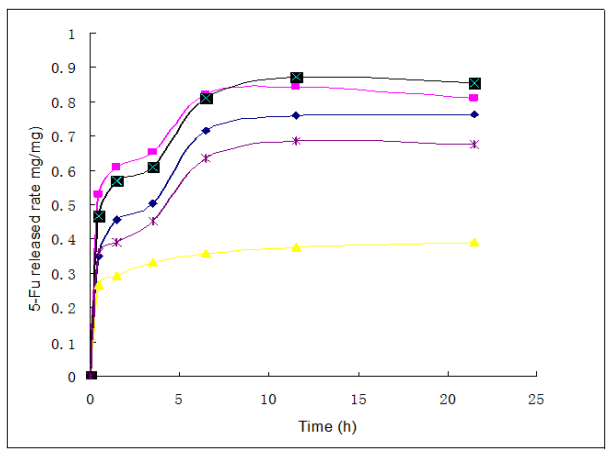

Figure 6. 5-Fu released rate from CS microparticles, with different stirring rate(rpm): $\bullet$ $650 ;(\bullet) 750 ;(\Lambda) 850$; (×)950; (*)1050

\section{Conclusions}

In this work, the CS microparticles are impregnated with 5-Fu by the ionic crosslinking method using TPP as crosslinker in order to develop hydrophilic drug release systems. The drug loading and release behavior of the 5-Fu loaded CS microparticles are investigated. The rusults show that, this method is simple and reproducible while drug release performance in vitro show that the microparticles have obviously sustained release effect.

\section{Acknowledgments}

This research is supported by the Doctoral Fund of Dalian University.

\section{References}

1. F. Casale, R. Canaparo, L. Serpe, E. Muntoni, et al, Pharm. Res. 50, 173-179(2004)

2. M. Simeonova, R. Velichkova, G. Ivanova, V. Enchev, I. Abrahams, Int. J. Pharm. 263, 133140(2003)

3. M.V. Natu, M. Gil, H.C. Sousa, J. Supercrit. Fluids. 47,93-102(2008)

4. G.C. Pisanelli, C.L. Wilt, H.M. Pinedo, F. Franchi, P. Noordhuis, B.J.M. Braakhuis, J.A.M. Laar, G.J. Peters, Eur. J. Cancer, 31, 1517-1525(1995)

5. T. Shashank, M.N. Satish Kumar, P. Ashwari, Saudi. Pharm. J. 23,308-314(2015)

6. S.P. Zhan, C. Chen, Q.C. Zhao, W.J. Wang, Z.J. Liu, Ind. Eng. Chem. Res. 52, 2852-2857(2013)

7. Y.L. Wang, Y.P. Wang, J. Yang, R. Preffer. R. Dave, B. Michniak, Power Technol. 164, 94102(2006)

8. B. Avinash, J.S. Steven, L, Karen, Int. J. Pharm. 51, 87-92(2007) 